\title{
Características de movilidad de la población residente entre el medio urbano y rural de una zona metropolitana
}

\section{Population travel characteristics between the urban-rural continuum in a metropolitan area}

\author{
Saúl Antonio Obregón-Biosca
}

\author{
Facultad de Ingeniería-Universidad Autónoma de Querétaro, \\ Querétaro, México
}

\section{Resumen}

La expansión territorial de asentamientos irregulares en las zonas periurbanas de una zona metropolitana influye sobre la población residente en dichos ámbitos en sus patrones y características en sus desplazamientos. A partir del estudio de las características de movilidad en países desarrollados, el presente artículo tiene como objetivo analizar y comparar las características de los desplazamientos de la población en el continuo urbano-rural de una zona metropolitana en un país emergente como México. Empleando los datos de una encuesta origen-destino y mediante estadística descriptiva, los principales resultados muestran las diferencias en los patrones y tasas de desplazamientos de la población que reside dentro del continuo urbano-rural considerando como caso de estudio la Zona Metropolitana de Querétaro.

Palabras clave: Viajes metropolitanos, medios de transporte, motivos de viaje, población, economía emergente.

\section{Abstract}

On a metropolitan area, the urban sprawl influences the mobility patterns of the population living in the peri-urban areas and in their travel characteristics, such as increases in travel times. Through the mobility characteristics studied in developed countries, so the paper aims to analyze and compare the population trips characteristics in the urban-rural continuum of a metropolitan area in emerging country as Mexico. From a survey origin-destination and descriptive statistics, the main findings have shown the differences in the mobility pattern and trip rate, of the people that live in the urban-rural continuum considering as case study the Metropolitan Area of Querétaro.

Keywords: Metropolitans trips, mode to transport, reason to travel, population, emerging economy. 


\section{INTRODUCCIÓN}

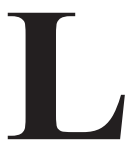

a dotación de transporte influye de forma indirecta como potencializador del crecimiento económico y social en un área dada, mientras su carencia también puede constituir una barrera (por costos, cobertura) que impida a potenciales trabajadores acceder al empleo (Obregón, 2010). En Henry (1998) se define la movilidad como la manera en que un individuo realiza un programa de actividades en sus dimensiones espaciales y temporales. Así, un viaje responde a cierto motivo utilizando un determinado medio en un momento temporal específico. En Ortúzar y Willumsen (2008) se define el concepto de movilidad como una medida de la facilidad o dificultad de realizar desplazamientos de un origen a un destino específico, mientras que en Giacobbe et al. (2009) se menciona que ésta cumple un rol muy importante en el análisis del desarrollo urbano, permitiendo una doble lectura: puede ser entendida en términos de la facilidad o dificultad con la que las personas acceden a lugares o también se puede entender en términos de la posibilidad o imposibilidad de acceder a servicios y lugares.

En las zonas metropolitanas se genera una expansión territorial de asentamientos irregulares en sus zonas periurbanas, en donde coexisten localidades de tipo urbano y rural, en las cuales se presentan procesos de degradación física y social. Dicha expansión territorial induce a alcanzar un número de destinos más dispersos y alejados del hogar para realizar un mayor número de actividades (Gutiérrez y García, 2005). La movilidad se materializa a nivel espacial en los desplazamientos generados producto de las necesidades cotidianas de los individuos. Mientras que los desplazamientos son consecuencia de la ubicación del hábitat y de la necesidad de realizar las actividades cotidianas, su distribución en el área urbana da lugar a la separación entre los puntos de origen y destino, por lo anterior un sistema de transporte en una ciudad debe de resolver las necesidades principales de su población en forma sostenible (Valero, 1984 y Pardo, 2005). La accesibilidad es un factor clave en la movilidad, y se define como la disponibilidad que un ciudadano tiene para movilizarse, y de esta manera satisfacer sus necesidades.

En García (2008) se sugiere que el análisis de la movilidad en la expansión urbana debe de tener en cuenta no sólo el número de viajes, sino también los tiempos de duración de cada viaje y sus distancias de recorrido, 
reconociendo que los viajes a gran distancia son menos frecuentes que los viajes a corta distancia. Dentro de la estructura y la funcionalidad de una zona metropolitana, se resalta la importancia que presentan los desplazamientos, la infraestructura viaria, y la distinción entre una localidad urbana o rural (Song, 1992; García, 2010 y Bazant, 2010). En los análisis de movilidad se ha reportado un mayor número de trabajos de investigación en países desarrollados enfocados al análisis los desplazamientos producidos en el periurbano (Harvey, 1994; Millward y Spinney, 2009 y Millward y Spinney, 2011), destacando la investigación reportada en Pucher y Renne (2005), que analiza las longitudes de viaje, la elección modal y el propósito de viaje, pero no prueba de diferencias significativas entre las áreas periféricas.

El caso de estudio más reciente es el de Millward y Spinney (2011), en la región Halifax, Nueva Escocia, en el cual se analizan las características de los desplazamientos de la población, delimitando regiones con base a la distancia de viaje por vialidades, partiendo del núcleo central de población. En dicho estudio se muestran patrones diferenciados de las características de movilidad de la población entre el entorno urbano y el entorno rural. Por lo anterior, partiendo de la metodología empleada en Millward y Spinney (2011) con relación a la delimitación de las regiones considerando la distancia de viaje, la presente investigación tiene como objetivo determinar las características de los patrones de movilidad de la población en dichas regiones, bajo la siguiente hipótesis de partida: se presentan características disímiles y definidas en los patrones de movilidad de la población residente entre el medio urbano y el rural exterior de una zona metropolitana. La investigación compara los principales resultados obtenidos frente al caso de estudio en la región de Halifax, con el objetivo de determinar coincidencias y diferencias entre un país desarrollado y uno emergente.

\section{MARCo de REFERENCIA}

En el presente apartado se discute el concepto de una Zona Metropolitana (ZM), su estructura interna y cómo dicha estructura influye en los desplazamientos. Dentro de los elementos de dicha estructura se discute el concepto de dispersión urbana, sus acepciones, el cómo se presenta el fenómeno, por qué los individuos eligen vivir ahí, así como las diferencias de dicho fenómeno en economías desarrolladas y emergentes. Por último, se exponen las características de los desplazamientos de la población en entornos periféricos y los parámetros que se analizan. 


\section{La zona metropolitana}

En una ZM se presenta la mayor concentración población, dinámica y economía dentro de una región, en ellas se genera la expansión territorial y los asentamientos irregulares en sus zonas periurbanas (Obregón y Bueno, 2015). En las ZM coexisten localidades urbanas y rurales, siendo una de las dificultades el distinguir donde inicia una y dónde termina otra, por lo que Bazant (2010) destaca que podemos encontrar una localidad totalmente urbanizada y presenciar a su alrededor características totalmente rurales.

Dicha expansión se presenta generalmente en dirección lineal siguiendo una infraestructura vial o radial alrededor de la ciudad lo que ha influido a la formulación de dos modelos teóricos de estructura y funcionalidad de una ZM: el modelo monocéntrico y el policéntrico. De acuerdo con Obregón y Bueno (2015) en el modelo monocéntrico, el núcleo central de la zona es el que ejerce el principal dominio, mientras en el modelo policéntrico además del núcleo central, existen subcentros en donde se distribuyen actividades. En Escolano y Ortiz (2005) se define el modelo mono-policéntrico, como el estado de transición entre uno y otro, y se caracteriza por mantener en el núcleo central cierto dominio, mientras que el desarrollo de otros subcentros extiende su influencia a toda la ciudad y la existencia de otros menores que ejercen su influencia en áreas más reducidas.

La forma y funcionalidad de dichas estructuras dentro de una ZM tienen relación directa sobre la longitud de los desplazamientos de la población, en este sentido, Song (1992) observó cómo una estructura de subcentros minimiza la longitud de los desplazamientos, y concluye que resulta deficiente el esquema monocéntrico, el cual influye en los patrones de los desplazamientos e induce su incremento (Dupuy, 1995). Lo anterior, se pone de manifiesto que la estructura de una Zona Metropolitana influye en la movilidad de la población.

\section{El concepto de dispersión urbana}

El proceso gradual de expansión fuera de la urbanización, ha generado el término "dispersión urbana" (Bruekner y Fansler, 1983), que involucra al transporte y se caracteriza por transitar a través de un suelo vacante entre un área y otra (Zhang, 2004 y Hamidi y Ewing, 2014). La dispersión urbana es un concepto con dificultad en su definición, pero en Bhatta (2010) se expone que el consenso general "se caracteriza por un patrón no planificado y desigual en su crecimiento, impulsado por una multitud de procesos y que conduce a la utilización ineficiente de los recursos". Algu- 
nos efectos indeseables al presentarse este fenómeno, incluyen la perdida de suelo agrícola y la reducción del capital social (Brueckner y Largey, 2008 y Putnam, 2000). Por lo anterior, Rahman et al. (2008) sostiene que estas zonas periféricas se caracterizan por un desarrollo urbano adverso que interfiere con el entorno urbano, lo cual no es ni una situación urbana aceptable ni una situación adecuada para un entorno rural-agrícola. En este contexto, Zasada et al. (2011) y Haller (2014) coinciden que el proceso de periurbanización se caracteriza por los asentamientos más allá de las zonas urbanas y dicha dispersión puede contribuir a satisfacer la necesidad de crecimiento de las zonas urbanas para dar cabida a una parte de un número creciente de habitantes.

Cabe señalar que el término periferia presenta dos acepciones, la geográfica y la social. La primera, Hiernaux y Lindon (2004) la definen como parte del proceso de expansión de la ciudad sobre zonas colindantes o periféricas a la ciudad de vocación rural, mientras la segunda, en Blanco et al. (2012) se precisa como territorios que concentran procesos de degradación física y social en consecuencia a la concentración de problemas, desatención de los poderes públicos y desinterés-marginación del capital privado. Empero, no todas las periferias urbanas son espacios de marginación y no todos los espacios de carencia social son periféricos, por ello, en Díaz (2012) se expone que no todas las periferias urbanas presentan las mismas características, y que cada una refleja diversas condiciones.

En la acepción geográfica, para Ravetz et al. (2013), el periurbano es a menudo una zona de urbanización caótica que conduce a la dispersión, mientras que en Friedmann (2011) la región periurbana es una "zona de encuentro, el conflicto y la transformación que rodean a las grandes ciudades". Estas zonas se desarrollan, por un lado, a través de nuevos asentamientos que proporcionan una mejor vivienda o una más asequible para las personas que viven en las zonas urbanas, y por el otro, a través de los asentamientos rurales que evolucionan a un carácter más urbano y que exigen una mejor conectividad a las áreas urbanas (Webster, 2002).

Dichos desarrollos periféricos son el resultado de características económicas y sociales, como por ejemplo: suelo más barato, terrenos con una mayor extensión y vivienda asequible en los suburbios; además, la búsqueda de tranquilidad, y a veces la construcción de infraestructuras específicas designadas para promover los asentamientos en las zonas periféricas, pueden detonar la dispersión urbana (Harvey y Clark, 1965; Nechyba y Walsh, 2004; Allard, et al., 2014 y Zhang, 2010). No obstante, la población al elegir su residencia en dichas zonas normalmente subestima los mayores 
costos de transporte que implica el desplazamiento al núcleo central (Newman y Kenworthy, 1988; Nicolas, et al., 2003; Rouge, 2005; Banister y Hickman, 2006; Allard et al., 2014 y Obregón et al., 2016).

La dispersión urbana se presenta indistintamente a su nivel de desarrollo en diversos países, y tal fenómeno demográfico tiene causas y efectos distintivos para los países desarrollados y emergentes. En economías desarrolladas, existe la sub-urbanización de lujo, que se caracteriza por viviendas de alto costo, mientras en economías emergentes el fenómeno de la periurbanización es doble, pues va en función de la disponibilidad de la infraestructura y de los servicios públicos. Es decir, por un lado, hay zonas residenciales, pero en el otro, hay asentamientos irregulares, en parte como resultado del nivel económico, pues al tener un modesto ingreso la migración interna se instala en los ámbitos periféricos (Da Gama-Torres, 2008; ONU, 2008). Otro aspecto distintivo en dichos países consiste en desarrollos masivos periurbanos dirigidos a potenciales propietarios que pueden pagar una hipoteca. Estas urbanizaciones ofrecen pequeñas viviendas económicamente asequibles de menos de $50 \mathrm{~m}^{2}$, situadas en el periurbano. En los países emergentes como México, otro proceso se ha producido en las afueras de los centros urbanos, que consiste en la construcción de parques industriales, que incorporan empresas principalmente con capital extranjero, las cuales crean puestos de trabajo, pero sus trabajadores están vinculados con los núcleos centrales, ya sea del mismo municipio o un municipio vecino (Webster y Muller, 2002).

A pesar de las diferentes causas y los efectos involucrados, el crecimiento y externalidades de los ámbitos periurbanos se han convertido en un objeto de estudio tanto en las economías desarrolladas como emergentes. Por ejemplo, en economías desarrolladas, Norman et al. (2006) hacen hincapié en cómo los desarrollos suburbanos de baja densidad consumen más energía y generan mayores emisiones de gases de efecto invernadero en comparación con los desarrollos urbanos, mientras en economías emergentes como México y Brasil, Sperandelli et al. (2013) describen cómo las externalidades de la dispersión urbana incluyen la devastación de zonas verdes y el uso generalizado de los vehículos particulares, en un contexto de planificación ineficiente en el uso del suelo, y por ello, cómo la mancha urbana continúa exigiendo más cambios de suelo. Tanto en los países desarrollados como emergentes los efectos de la expansión se han evaluado en términos del consumo adicional de combustible, como en Baur et al. (2014) y en Obregón et al. (2016), no obstante, se han realizado un mayor número de investigaciones en países desarrollados enfocadas a analizar los 
desplazamientos producidos en el periurbano. Por lo anterior, Millward y Spinney (2011) sostienen que, aunque se han reportado resultados que examinan las diferencias en el urbano-suburbano (como en Cervero y Gorham, 1995, Goudie, 2002, en la elección modal y en Chen y McKnight, 2007, en los tiempos de viaje), no se han examinado de manera satisfactoria las diferencias en el comportamiento de los desplazamientos a lo largo del continuo rural-urbano, aportando dichos autores resultados que serán comparados y discutidos en el presente artículo.

\section{La movilidad en ámbitos periféricos}

Los desplazamientos inducidos por motivo de trabajo y estudio que envuelven a los núcleos centrales y a las zonas periféricas, se han convertido en un problema social y económico a analizar. Sobrino (2007) afirma que la localización residencial está influenciada por la localización del lugar de trabajo, cabe señalar que dicha relación causa-efecto no se define con claridad, es decir, si el residente primero obtuvo el puesto de trabajo y luego decidió su lugar de residencia, o viceversa. En la Zona Metropolitana de Querétaro Obregón et al. (2016) reportan que los residentes de las áreas periféricas decidieron la localización de su residencia en primer lugar por el ambiente y la tranquilidad, en segundo por el precio del suelo, el tercero por razones familiares y en cuarto lugar por razones de trabajo.

En el inevitable incremento en la movilidad inducido por la dispersión urbana, no debe considerarse únicamente el número de desplazamientos, pues tal como se sugiere en García (2008), también se deben analizar los tiempos de viaje y sus distancias, ya que se infiere que serán menos frecuentes los viajes a grandes distancias. Así, los cambios dimensionales dentro de las áreas metropolitanas en dispersión originan adaptaciones territoriales y sociales, desarrollando un creciente número de destinos fuera del núcleo central con una gama más amplia de actividades. Tal como lo plantea Ascher (2004), el desarrollo del transporte y las comunicaciones alarga las distancias diarias recorridas, multiplicando las opciones de movilidad para una gran parte de la población en todos los aspectos de su vida: el lugar de residencia, la localización de actividades y trabajos, así como para propósitos de socialización. Sin embargo, como ya se expuso, las externalidades negativas de la dispersión urbana no sólo se centran a la disminución del suelo agrícola, sino además de las ya referidas, en el cumplimiento de las necesidades de movilidad de la población periurbana que viaja a los núcleos urbanos (Lucatelli, 2011). 


\section{Caso de estudio}

La Zona Metropolitana de Querétaro se define en SEDESOL, CONAPO e INEGI (2012) considerando características urbanas y de viajes intermunicipales por motivos de trabajo, entre otras. En México el fenómeno de metropolización comenzó a presentarse en los años 50 del siglo pasado, y en 1970 se establecen oficialmente 12 zonas metropolitanas (Unikel et al., 1976), alcanzando $59 \mathrm{ZM}$ en el 2010. La ZMQ está conformada por cuatro municipios: Querétaro, Corregidora, El Marqués y Huimilpan (Figura 1), dichos municipios fueron definidos de acuerdo a los criterios establecidos en SEDESOL, CONAPO e INEGI (2012).

En la Zona Metropolitana de Querétaro se muestran mayores ritmos de crecimiento demográfico (2.4 por ciento anual) que otras zonas metropolitanas similares en México alcanzando 1,097,025 habitantes en el año 2010 tal y como se observa en la Figura 2, mientras que su parque vehicular pasó de 577,764 vehículos registrados en el año 2011 a 635,133 en 2013, distribuyéndose en 65.8 por ciento de automóviles, 29.3 por ciento de camiones, 3.1 por ciento de motocicletas y 0.47 por ciento de autobuses (Obregón et al., 2015a).

El ritmo del crecimiento demográfico y de la expansión territorial se expresan, en mayores longitudes de viajes, mayores costos por concepto de movilidad y mayores emisiones polucionantes, tal como se reporta en Obregón et al. (2016). Sin embargo, no son los únicos efectos debido a este crecimiento del territorio ocupado por las actividades urbanas, ni a su equivalente incremento en tiempo de viaje, al desgaste vehicular, o la inferencia que de ahí puede derivarse que muy probablemente las situaciones de congestión, contaminación y accidentalidad hayan empeorado. Pero para dar una primera aproximación a lo planteado es necesario analizar y cuantificar las prácticas de movilidad en ámbitos periféricos dependiendo de su distancia al núcleo central, tal como se plantea en Millward y Spinney (2011) y objeto de la presente investigación.

\section{Metodología}

Para el proceso de recopilación de datos, se delimitaron las diferentes áreas poblacionales considerando el área geoestadística básica (AGEB) definida por el Instituto Nacional de Estadística y Geografía de México (INEGI, 2010) como la unidad básica del marco geoestadístico nacional. 
Características de movilidad de la población residente entre el medio urbano y rural ... /S.A. OBREGÓN BIOSCA

Figura 1: La Zona Metropolitana de Querétaro



Fuente: elaboración propia. 


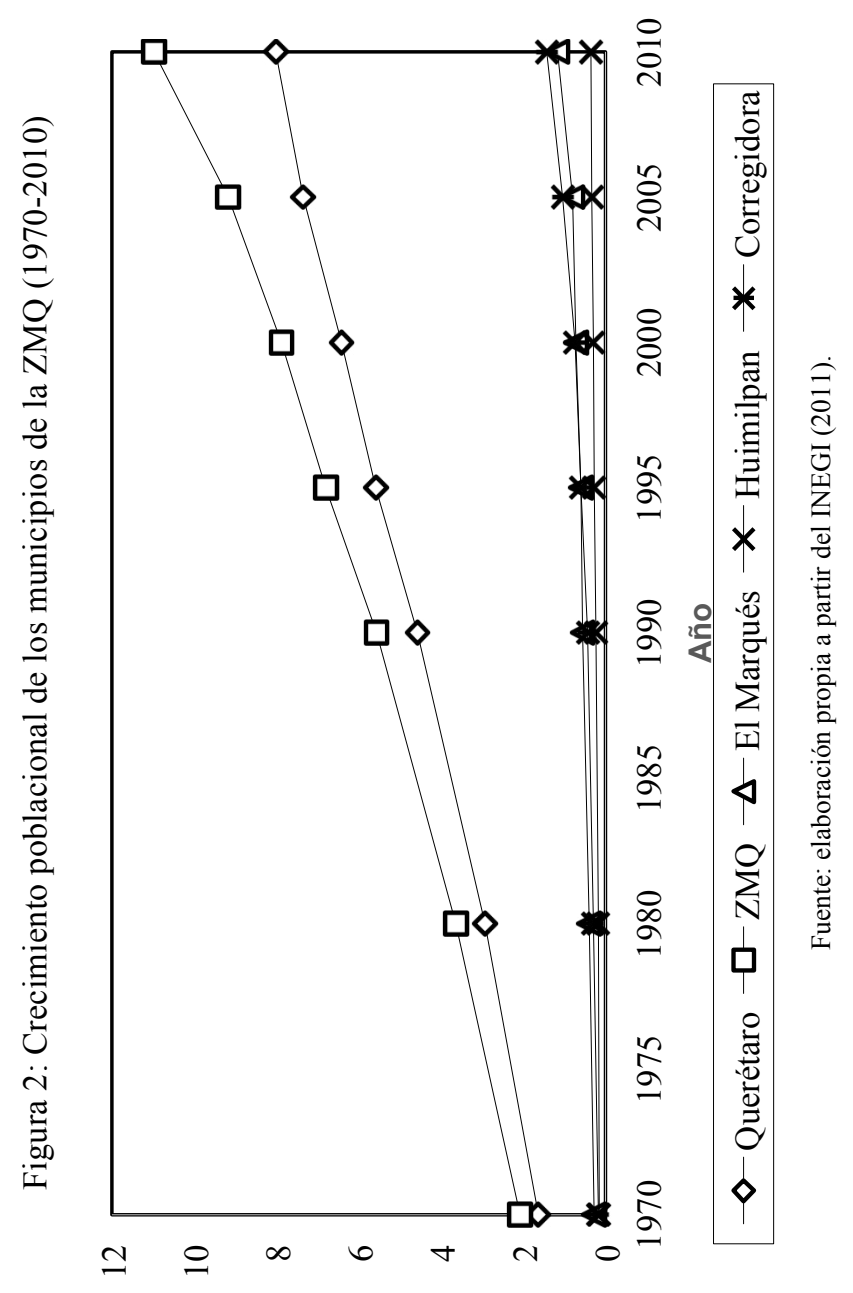

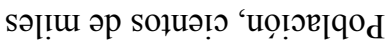


Considerando el criterio de estratificación mostrado en Smith (1979) y que se emplea en los estudios Origen-Destino, se parte de que la división de estratos de población debe realizarse de tal manera que cada uno de sus elementos sean homogéneos con respecto a las variables de estudio. Para dicha división de estratos se consideró el AGEB. Con relación al tamaño de la muestra, se siguieron las recomendaciones expuestas en Bruton (1985), Ibeas et al. (2007) y en Ortúzar y Willumsen (2008), obteniendo el tamaño de la muestra de hogares para aplicar el cuestionario, resultando lo siguiente: 3,849 hogares en la Zona Metropolitana de Querétaro, de los cuales 1,030 hogares se localizan en la Zona Periférica. Su aplicación se realizó entre los meses de mayo y septiembre del año 2012, exceptuando el período vacacional y los días festivos. El proceso detallado del muestreo se puede consultar en Obregón y Bueno (2015) y Obregón et al. (2016). Los elementos que se consideraron en el cuestionario proporcionaron la información básica para caracterizar la generación y la atracción de los viajes en la zona geográfica de estudio; los tiempos de viaje; los modos de transporte; la distribución horaria; y las características socioeconómicas de la población residente. Para lograr el objetivo del presente trabajo, se empleó estadística descriptiva como herramienta para analizar las características socioeconómicas de la población residente en cada área, los motivos del viaje y el número y la cantidad de usuarios de cada modo de transporte, incluyendo los respectivos tiempos de viaje.

Las variables de movilidad de la población en el ámbito periférico de la Zona Metropolitana de Querétaro, se analizan bajo el esquema de zonificación que se propone en Millward y Spinney (2011) donde se aborda conceptualmente la zonificación de la franja rural-urbana considerada en Wehrwein (1942), Pryor (1968), Furuseth y Lpping (1999), Coppack et al. (1988) y en Bryant et al. (2000). En dichos trabajos se delimitan las zonas operativamente, considerando la forma urbana y los vínculos de desplazamientos a la zona urbana. Con base a dichas consideraciones se definen cinco zonas: i) zona urbana: el continuo urbano ii) suburbios: zona contigua urbanizada dentro de los límites de servicios urbanos, se consideran todas las áreas dentro de cinco kilómetros de distancia por vialidades desde el límite externo de la zona urbana iii) cinturón suburbano interior (CSI): todas las otras áreas dentro de 25 kilómetros de distancia por carretera desde el límite externo de la zona urbana. En Millward y Spinney (2011) se expone que dicha zona es afectada por una gran cantidad de desarrollos inmobiliarios, y la mayoría de sus residentes trabajan en la zona urbana iv) cinturón suburbano exterior (CSE): áreas ubicadas entre 25 y 50 kilóme- 
tros de distancia por carretera desde el límite externo de la zona urbana. En dicha región se presenta menor número de desplazamientos a la zona urbana y se depende más de sus recursos locales v) rural remoto: zonas más allá de los 50 kilómetros por carretera desde el límite de la zona urbana. Para nuestro caso de estudio se consideran cuatro de las cinco áreas mencionadas: i) zona urbana: la conurbación, ii) suburbios: todas las áreas dentro de cinco kilómetros de distancia por vialidades desde el límite externo de la zona urbana iii) cinturón suburbano interior: todas las áreas dentro de 25 kilómetros de distancia por carretera desde el límite externo de la zona urbana, y iv) cinturón suburbano exterior: zonas ubicadas más allá de los 25 kilómetros por carretera desde el límite de la zona urbana. A diferencia de lo que se reporta en Millward y Spinney (2011), no se considera la zona rural remota, debido a que la Zona Metropolitana de Querétaro no presenta una extensión superior a 50 kilómetros. Las áreas urbanas y rurales que abarcan cada una de las cuatro zonas se muestran en la Figura 3, obtenido a través de un grafo georreferenciado y empleando el software Transcad Ver. 7 considerando la distancia sobre una vialidad (representadas por arcos) a cada una de las poblaciones.

\section{Resultados Y Discusión}

Demográficamente, al considerar la zonificación expuesta, se observa que en el CSE reside el mayor porcentaje de personas mayores a 55 años, y de forma opuesta el grupo menor a 15 años refleja el mayor porcentaje en los suburbios. La Tabla 1 muestra dichas características socioeconómicas en cada una de las zonas, en donde la mayor tasa de hombres se observa en los suburbios, en dicha zona se aprecia la mayor tasa de trabajadores y estudiantes, y conforme se incrementa la distancia a la conurbación dicha tasa disminuye y de forma contraria, a mayor distancia la tasa de personas que no tienen ocupación se incrementa, de igual manera que, la tasa de población dedicada al hogar. Lo anterior no coincide con lo que se identifica en Millard y Spinney (2011), donde se reportan mayores tasas a mayor cercanía con la conurbación, en ese sentido hacen referencia a Davies y Murdie (1993) sobre que lo expuesto es un patrón social identificado en los estudios urbanos, empero en nuestro caso de estudio no se refleja, posiblemente por la influencia de las dinámicas laborales y porque es una población más joven la que reside en dicha zona, con respecto al resto. 
Figura 3: Asentamientos urbanos - rurales en la Zona Metropolitana de Querétaro

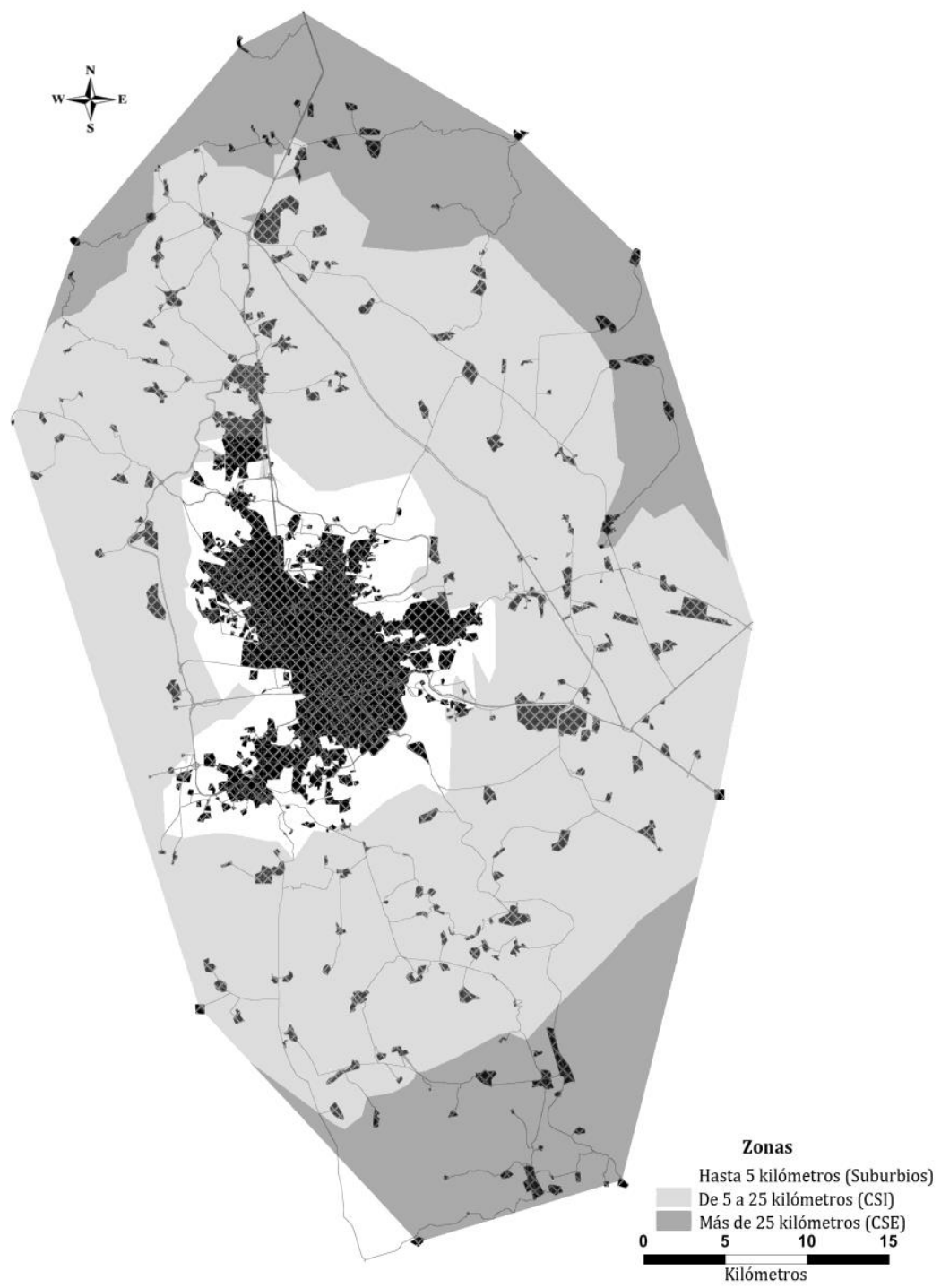

Fuente: elaboración propia. 
Tabla 1: Características socioeconómicas de las localidades en cada zona por grupo demográfico (en porcentaje)

\begin{tabular}{rrrrr}
\hline Encuestados $(n)$ & Total & Suburbios & $\begin{array}{r}\text { Cinturón } \\
\text { suburbano interior }\end{array}$ & $\begin{array}{r}\text { Cinturón } \\
\text { suburbano exterior }\end{array}$ \\
\hline & 100.0 & 20.28 & 71.88 & 7.84 \\
& $(3393)$ & $(688)$ & $(2439)$ & $(266)$
\end{tabular}

Grupo de edad

Menos de 15 años

$16.7 \quad 17.7$

16.6

12.4

15 a 34

40.8

39.5

41.4

41.0

35 a 54

30.4

34.0

29.3

55 o más

12.1

8.7

29.4

17.3

Género

Mujer

49.1

46.7

49.9

48.5

Actividad principal

Trabaja

46.9

48.8

46.8

46.8

Estudia

22.8

24.3

22.4

16.7

Hogar

22.2

18.9

23.0

26.2

Jubilado-pensionado

2.1

3.6

1.6

2.7

Sin ocupación

$6.0 \%$

4.4

6.2

7.6

Licencia de conducir

Sí

23.9

29.1

22.4

19.0

Educación

Grado universitario

$$
6.3
$$

8.1

5.9

1.1

Ingreso familiar en salarios mínimos diarios

Menos de 2

10.5

55.1

20.3

14.1

De más de 6 a 10

6.6

8.7

27.6

De 2 a 6

50.5

54.0

21.4

15.9

15.6

1.2

6.5

8.9

Primario

5.5

38.0

37.6

39.8

31.7

Terciario

56.5

61.2

Fuente: elaboración propia. 
Otras características son, que la población cuente con un grado de educación universitario y con licencia de conducir, su tasa disminuye conforme la distancia a la conurbación se incrementa. Con relación al ingreso por familia, los resultados muestran que el CSE es la zona que refleja el mayor porcentaje de familias con menor ingreso económico, mientras los suburbios muestran mayor tasa en las familias con ingresos superiores a seis salarios mínimos diarios (en el año de aplicación del cuestionario). Tal como se planteó, en la zonificación se describe cómo la lejanía a la conurbación influye a que se presenten características rurales en las localidades. Lo anterior se comprueba cuando se observa que el CSE exhibe una mayor tasa de población ocupada en actividades primarias. El CSI muestra la mayor tasa de población ocupada en el sector secundario de las tres zonas. Lo anterior se debe a que cinco parques industriales (Parque Industrial Querétaro, La Cruz, Aeropuerto, Bernardo Quintana y El Marqués) se ubican en dicha franja.

\section{Desplazamientos, tiempos y vehículos}

La tasa promedio de desplazamientos por persona disminuye conforme la población reside a mayor distancia del núcleo urbano. Tal como se muestra en la Tabla 2, el CSE muestra la menor tasa de desplazamientos. Con respecto al tiempo de duración del viaje, se considera el viaje principal (por movilidad ocupacional) como el promedio por persona. Éstos tiempos son similares tanto en los suburbios como en el CSI, mientras en el CSE el tiempo promedio de viaje se incrementa en más de diez minutos con respecto a las otras dos zonas. Es decir, la población que reside en el CSE es aquella población que invierte mayor tiempo en promedio en sus desplazamientos. Se supone que los tiempos de viaje en CSI serían mayores con respecto a los tiempos de viaje en los suburbios. Sin embargo, la dotación de servicios y puestos de trabajo (principalmente en el sector secundario) en dicha zona, influyen en una menor participación hacia el núcleo central.

En cuanto a la tenencia de vehículos particulares en el hogar, se observa que, tanto en el CSI y en el CSE se ubican las tasas más altas de hogares que no cuentan con vehículo propio, pero en el CSI se tiene la mayor tasa de dos o más automóviles en el hogar de las tres zonas. Lo anterior responde a que en dicha área se presenta la tasa más alta de ingreso económico por familia (más de diez salarios mínimos) de las tres zonas analizadas. Con relación a las características de los vehículos, la mayor tasa de vehículos con seis y más cilindros se refleja en el CSE, lo que concuerda con la potencia del vehículo necesaria para realizar actividades del sector prima- 
rio. De igual manera, el parque vehicular de mayor antigüedad se localiza en dicha zona.

Tabla 2: Información de desplazamientos por localidad en cada zona

\begin{tabular}{lrrrr}
\hline Encuestados $(n)$ & & & $\begin{array}{r}\text { Cinturón } \\
\text { suburbano } \\
\text { interior }\end{array}$ & $\begin{array}{r}\text { Cinturón } \\
\text { suburbano } \\
\text { exterior }\end{array}$ \\
\hline & $n=3393$ & $n=688$ & $n=2439$ & $n=266$ \\
Desplazamientos promedio por persona & 2.321 & 2.34 & 2.312 & 2.206 \\
$\begin{array}{l}\text { Tiempos de viaje } \\
\text { Tiempo promedio de viaje por persona }\end{array}$ & & & & \\
en su principal desplazamiento (min) & 38.18 & 37.88 & 37.28 & 47.09 \\
Tiempo promedio por desplazamientos & 37.58 & 37.55 & 36.53 & 47.42 \\
y persona (min) & & & & \\
Automóvil en el hogar & & & & \\
Sin automóvil & $45.9 \%$ & $43.4 \%$ & $47.1 \%$ & $46.9 \%$ \\
Un automóvil & $41.7 \%$ & $48.1 \%$ & $39.3 \%$ & $46.9 \%$ \\
Dos o más & $12.3 \%$ & $8.5 \%$ & $13.5 \%$ & $6.2 \%$ \\
Cilindros de los vehiculos & & & & \\
4 cilindros & $65.5 \%$ & $69 \%$ & $63.3 \%$ & $56.5 \%$ \\
6 cilindros & $28.0 \%$ & $21 \%$ & $31.2 \%$ & $30.4 \%$ \\
8 cilindros & $6.6 \%$ & $10 \%$ & $5.5 \%$ & $13.0 \%$ \\
Antigüedad de vehículos & & & & \\
Menos de 5 años & $15.2 \%$ & $14.7 \%$ & $16.9 \%$ & $0.0 \%$ \\
De 5 a 10 años & $17.5 \%$ & $22.1 \%$ & $16.7 \%$ & $11.1 \%$ \\
De 10 a 20 años & $46.4 \%$ & $44.1 \%$ & $44.7 \%$ & $68.9 \%$ \\
Más de 20 años & $21.0 \%$ & $19.1 \%$ & $21.6 \%$ & $20.0 \%$ \\
\hline
\end{tabular}

Fuente: elaboración propia.

\section{Viajes por grupo de actividad}

En la Tabla 3 se muestra la tasa promedio de viajes por persona y por día dependiendo del motivo del viaje. En ella se observa que la tasa de desplazamientos por motivo de trabajo se incrementa (mínimamente) conforme la distancia al núcleo central aumenta. Sin embargo, la tasa de deslazamientos por viajes relacionados al trabajo es mayor en los suburbios. Lo anterior indica que los trabajadores que residen en los cinturones suburbanos, en su jornada y características laborales, les es necesario realizar menor número de desplazamientos derivados de su actividad. 
Tabla 3: Tasa de viajes por grupo de actividad

\begin{tabular}{|c|c|c|c|c|}
\hline Motivo del viaje & Total & Suburbios & $\begin{array}{r}\text { Cinturón } \\
\text { suburbano } \\
\text { interior } \\
\end{array}$ & $\begin{array}{r}\text { Cinturón } \\
\text { suburbano } \\
\text { exterior }\end{array}$ \\
\hline Al y desde trabajo remunerado & 0.83 & 0.82 & 0.83 & 0.84 \\
\hline Relacionado al trabajo remunerado & 0.03 & 0.05 & 0.02 & 0.03 \\
\hline Educación & 0.29 & 0.33 & 0.28 & 0.29 \\
\hline Compra de bienes y & 0.20 & 0.18 & 0.22 & 0.10 \\
\hline Salud & 0.07 & 0.05 & 0.07 & 0.11 \\
\hline Deporte & 0.02 & 0.03 & 0.02 & 0.00 \\
\hline Recreación & 0.04 & 0.05 & 0.04 & 0.04 \\
\hline Asuntos personales y familiares & 0.25 & 0.30 & 0.23 & 0.23 \\
\hline Otros & 0.06 & 0.05 & 0.06 & 0.04 \\
\hline
\end{tabular}

Fuente: elaboración propia.

Se destaca la alta tasa de viajes por persona y por motivo de estudio en los suburbios, lo cual se liga a que es en la zona donde reside el mayor porcentaje de estudiantes y de población joven de las tres zonas. Además, el que refleje una tasa superior se induce a estudios de tipo superior, en donde se presentan diferentes horarios, y por ello influye en una mayor tasa de desplazamientos. Con relación a la tasa de viajes por motivo de compra de bienes y de servicios, ésta es superior en el CSI, seguido de los suburbios, esto producto del mayor ingreso familiar en dichas zonas, mientras que la distancia al núcleo central y el poder adquisitivo de las familias en el CSE, influyen en el decremento en la tasa de viajes. Empero, en dicha zona se presenta la tasa de viajes promedio, por motivo de salud, más alta. Lo anterior deriva del mayor porcentaje de personas de edad avanzada que residen en el CSE.

La tasa de desplazamientos por motivo de la realización de deportes es mayor en los suburbios, seguido del CSI, debido a la dotación de infraestructura y de servicios en dichas zonas, mientras que en el CSE no se reportaron desplazamientos. La tasa de viajes por asuntos personales y familiares es superior en los suburbios con respecto al resto de zonas. $\mathrm{Su}$ ubicación "central", influye de manera directa en el incremento de este motivo de viajes.

En cuanto al tiempo promedio empleado para los desplazamientos realizados por cada tipo de actividad, en la Tabla 4 se muestra que conforme la zona se aleja del núcleo central, los tiempos empleados en los desplazamientos por motivo de trabajo se incrementan. No obstante, el tiempo promedio que se emplea para viajes escolares disminuye conforme se aleja 
del núcleo central. Lo anterior producto de la dotación de puestos escolares en las localidades de los cinturones suburbanos, y a la preferencia de la población de los suburbios para asistir a las escuelas particulares o públicas que se ubican en el núcleo central, induciendo mayor congestión vehicular en dicha zona.

Tabla 4: Duración promedio del viaje por tipo de actividad

\begin{tabular}{lrrrr}
\hline Motivo del viaje & Total & Suburbios & $\begin{array}{r}\text { Cinturón } \\
\text { suburbano } \\
\text { interior }\end{array}$ & $\begin{array}{r}\text { Cinturón } \\
\text { suburbano } \\
\text { exterior }\end{array}$ \\
\hline Al y desde trabajo remunerado & 39.27 & 37.92 & 38.28 & 52.76 \\
Relacionado al trabajo & 42.43 & 35.00 & 45.00 & 66.67 \\
remunerado & & & & \\
Educación & 32.34 & 37.05 & 30.72 & 29.59 \\
Compra de bienes y servicios & 36.32 & 34.40 & 35.85 & 53.64 \\
Salud & 53.19 & 50.26 & 52.70 & 60.83 \\
Deporte & 15.00 & 11.67 & 16.88 & \\
Recreación & 36.33 & 33.94 & 37.54 & 45.33 \\
Asuntos personales y familiares & 27.80 & 29.95 & 25.09 & 37.72 \\
Otros & 33.17 & 30.26 & 34.28 & 30.00 \\
\hline
\end{tabular}

Fuente: elaboración propia.

En cuanto a los desplazamientos motivados por la compra de bienes y servicios, de deporte, de recreación y de salud, se observa que a mayor lejanía con el núcleo central se presenta mayor tiempo en los viajes. En estos motivos, el mayor tiempo empleado en el desplazamiento, presenta una relación inversa con la tasa promedio de viajes por persona, es decir, a mayor tiempo menor tasa de viajes, a excepción de los viajes por motivo de salud, donde el CSE refleja la mayor tasa de viajes y el mayor tiempo promedio empleado para realizarlos.

\section{Medios de transporte}

El medio de transporte empleado para realizar el desplazamiento en cada una de las zonas, así como la tasa de viajes promedio por persona se muestra en la Tabla 5. Los suburbios reflejan la mayor tasa de viajes realizados como conductor de vehículo, así como el mayor porcentaje de desplazamientos en automóvil. Conforme hay una mayor distancia del núcleo central, ambas tasas de viajes disminuyen. Se destaca, que en el CSI se reporta el mayor porcentaje de viviendas con automóvil. Sin embargo, el 
suburbano evidencia un uso más intensivo de dicho medio, por la mayor cercanía a los lugares donde las diversas actividades se desarrollan en el núcleo central.

Tabla 5: Porcentaje de desplazamientos por medio (a) y tasa media de viajes por persona (b)

\begin{tabular}{lrrrrrrrr}
\hline & \multicolumn{1}{c}{ Total } & \multicolumn{2}{c}{ Suburbios } & \multicolumn{2}{c}{ Cinturón } & \multicolumn{2}{c}{ Cinturón } \\
& \multicolumn{1}{c}{$\mathrm{a}$} & $\mathrm{b}$ & $\mathrm{a}$ & $\mathrm{b}$ & $\mathrm{a}$ & $\mathrm{b}$ & $\mathrm{a}$ & $\mathrm{b}$ \\
\hline $\begin{array}{l}\text { Automóvil } \\
\text { (conductor) }\end{array}$ & 24.0 & 2.56 & 27.3 & 2.80 & 23.5 & 2.50 & 17.0 & 2.27 \\
$\begin{array}{l}\text { Automóvil } \\
\text { (pasajero) }\end{array}$ & 9.0 & 2.34 & 9.4 & 2.43 & 8.8 & 2.34 & 9.8 & 2.13 \\
Autobús & 45.4 & 2.19 & 49.1 & 2.21 & 43.9 & 2.19 & 47.1 & 2.13 \\
Bicicleta & 1.6 & 2.13 & 0.7 & 2.00 & 1.9 & 2.10 & 2.3 & 2.67 \\
A pie & 20.0 & 2.33 & 13.4 & 2.25 & 22.0 & 2.35 & 23.9 & 2.31 \\
\hline
\end{tabular}

Fuente: elaboración propia.

Los viajes realizados en automóvil como pasajero presentan la mayor tasa en los suburbios, mientras que el mayor porcentaje de desplazamientos se presenta en el CSE. Es decir, en los suburbios, un mismo individuo realiza más viajes como pasajero, mientras que en el CSE un mayor porcentaje de personas viajan como pasajero en automóvil, pero individualmente realizan un menor número de viajes.

El autobús es el medio de transporte en el que se realiza el mayor porcentaje de desplazamientos en todas las zonas, siendo en los suburbios donde se observa el mayor porcentaje y a la vez, la mayor tasa de viajes por persona. Lo anterior se debe a una mayor cobertura y una mayor frecuencia del servicio en dicha área, con respecto al resto.

En cuanto a los medios no motorizados, la población del CSE muestra el mayor porcentaje de empleo y las mayores tasas de viajes en bicicleta con respecto al resto de zonas. Tal como se expuso, en dicha zona se presentan los menores ingresos familiares y la menor tenencia de vehículos, por lo que dicho uso se motiva por las características económicas de la población. En cuanto a los desplazamientos realizados a pie, el menor porcentaje de viajes y tasa de desplazamientos por persona se evidencia en los suburbios con respecto al resto de zonas, pues en la medida que nos alejamos del núcleo central, mayor el porcentaje de desplazamientos 
que se realizan a pie. La tendencia de desplazamientos empleando los dos medios activos en las tres zonas, es contraria a lo que se observa en Millard y Spinney (2011). Esto se debe a las características socioeconómicas de la población, tales como el ingreso, además de los atributos del medio, los cuales están directamente ligadas a la elección del medio de transporte en la zona periférica de Querétaro, tal como se reporta en Obregón et al. (2016) en el modelo de elección discreta estimado.

Con respecto a la duración promedio de viaje por cada medio de transporte, la Tabla 6 expone los tiempos empleados para cada una de las zonas. En los medios motorizados, en el automóvil y el autobús, los menores tiempos de viaje se presentan en los suburbios. Mientras que en los activos, como la bicicleta, los tiempos promedios son menores en el CSE. En los desplazamientos que se realizan a pie, los mayores tiempos promedios se presentan en el CSE y los menores tiempos se presentan en los suburbios. Así, la cercanía al núcleo central influye en los menores tiempos. Aunque en el CSE se observa el mayor tiempo promedio por desplazamiento, en dicha zona se tiene la tasa más alta de viajes a pie, lo cual corrobora que la característica económica de la población en dicha zona, influye de manera determinante en la utilización del medio de transporte para la realización de dichos desplazamientos. En el caso de estudio en Millard y Spinney (2011), la duración promedio de los desplazamientos a pie en el CSE es menor con respecto al resto de zonas, lo cual es contrario a lo reflejado en la zona periférica de Querétaro. En cuanto a los medios de transporte motorizados, los desplazamientos en autobús son los que presentan un mayor tiempo de duración promedio con respecto al automóvil. Lo anterior se debe principalmente a los tiempos de espera y tiempos de acceso a la parada, influyendo en duplicar el tiempo total de viaje (Obregón et al, 2015a).

Tabla 6: Duración del viaje por medio de transporte (minutos)

\begin{tabular}{lrrrr}
\hline & Total & Suburbios & $\begin{array}{r}\text { Cinturón } \\
\text { suburbano interior }\end{array}$ & $\begin{array}{r}\text { Cinturón } \\
\text { suburbano exterior }\end{array}$ \\
\hline Automóvil (conductor) & 27.09 & 25.47 & 27.10 & 37.78 \\
Automóvil (pasajero) & 27.60 & 23.88 & 26.25 & 47.97 \\
Autobús & 53.36 & 51.56 & 52.98 & 63.24 \\
Bicicleta & 28.77 & 25.00 & 30.15 & 21.25 \\
A pie & 18.98 & 20.66 & 17.46 & 28.69 \\
\hline
\end{tabular}

Fuente: elaboración propia. 
En la Tabla 7 se resumen los principales resultados sobre los desplazamientos por persona al día. En él, cada zona es definida con una nota de alto, medio y bajo para cada variable, con relación al valor medio de toda la muestra, indicando las diferencias entre cada una de las zonas, con el objeto de comparar los resultados obtenidos en este trabajo con los obtenidos en Millard y Spinney (2011) en el caso de estudio de la región de Halifax. La tasa promedio de viajes por persona en la ZM de Querétaro disminuye conforme la localidad se aleja del núcleo central, mientras que en la región de Halifax, se mantiene constante y disminuye a partir del CSE. Sobre los tiempos de viaje, en este trabajo no se refleja la variación entre los suburbios y el CSI, y en la región de Halifax los tiempos menores de viaje se presentan en los suburbios. La tasa de viajes por motivo de trabajo en la ZM de Querétaro se incrementa conforme se aleja del núcleo central, en la región de Halifax, las tasas más altas de viajes se reflejan en el CSI. Otra diferencia que se destaca es que en el CSI de la ZM de Querétaro se registran las mayores tasas de viajes por motivo de compras, y que en la región de Halifax este hecho se presenta en los suburbios. Con respecto a la duración promedio de los desplazamientos, no se observa gran variación en ambos casos de estudio. Siendo el CSE el que exhibe los tiempos más altos. La tasa de viajes en automóvil sí varía en ambos casos de estudio, pues mientras en la ZM de Querétaro la mayor tasa de viajes se presenta en los suburbios, en la región de Halifax se reporta en el CSI. Mientras los tiempos promedio de viaje se incrementan en ambos casos conforme aumenta la distancia al núcleo central.

\section{Conclusión}

Los resultados destacan las diferencias en las características de la movilidad de la población que reside dentro del continuo urbano-rural de una Zona Metropolitana, considerando como caso de estudio la de Querétaro, México. Dichas diferencias reflejan patrones disímiles entre el suburbano y el CSI, lo cual comprueba la hipótesis de partida.

En los contrastes realizados entre las zonas se destaca que a mayor separación del núcleo central los residentes tienden a viajar menos por la lejanía que les supone la ubicación de su residencia a las zonas de actividad y sus propias características económicas, es decir, la tasa promedio de viajes por persona disminuye conforme la localidad se aleja del núcleo central. En el mismo sentido, los mayores tiempos promedio de viaje en las tres zonas analizadas se presentan en el CSE. 





En cuanto a la tasa de desplazamientos por motivo de compra de bienes y servicios, se observa cómo ésta es menor en el CSE y es superior en el CSI con respecto a los suburbios, lo anterior está influenciado por la instalación de grandes superficies en el CSI, mientras que los tiempos promedio de viaje son similares en las tres zonas.

Las características socioeconómicas de la población tienen relación directa con la pertenencia de automóviles por hogar y a la vez con la posibilidad de realizar un mayor número de desplazamientos en medios motorizados, por ello, se manifiesta cómo la tasa de viajes promedio por persona al día en automóvil y en autobús disminuye conforme hay mayor distancia al núcleo central, mientras que en medios no motorizados, se incrementa la tasa al alejarse de la conurbación. En este sentido, los resultados clarifican dicha característica al observar que el CSE es la región con mayor aislamiento, actividades propias de una economía de entorno rural, presentan el mayor porcentaje de población desocupada, la mayor tasa actividades en el sector primario, el menor ingreso económico familiar, la menor tasa de población con estudios universitarios y tienden a minimizar los desplazamientos no esenciales (exceptuando por salud).

A diferencia del caso de estudio reportado en Millward y Spinney (2011), los suburbios de la Zona Metropolitana de Querétaro son los que presentan mayor participación de su fuerza laboral en el mercado de trabajo, mientras que la tasa más alta de viajes por motivo de trabajo, y con mayor duración, se presentan en el CSE, mientras en la región de Halifax el CSI presenta la mayor participación laboral, mayor tasa de viajes y mayores tiempos en los desplazamientos.

La presente investigación refleja que la dicotomía urbano/rural es suficiente como primera aproximación, para apreciar las variaciones entre las tasas de desplazamientos y el tiempo promedio empleado en los viajes entre las tres zonas analizadas. Lo anterior, puede auxiliar a diseñar políticas públicas en el sentido de la ordenación territorial, cobertura de transporte público y localización de servicios. En este sentido, los resultados muestran cómo la población del CSE requiere la cercanía de los servicios de salud. Con respecto a los medios no motorizados, estos reflejan mayores tasas de desplazamiento promedio en el CSI y en el CSE. Por lo anterior, políticas enfocadas al desarrollo de una red de carriles preferenciales potencializarán las tasas en el entorno suburbano.

En cuanto a las líneas de investigación futura, se propone una nueva clasificación basada en rangos de accesibilidad y cuadrantes, la cual puede proveer resultados con menor grado de generalización. 


\section{Agradecimientos}

Para la obtención de la información en la Zona Periférica de Querétaro se contó con el financiamiento del Consejo Nacional de Ciencia y Tecnología de México a través del contrato QRO-2010-C01-146269.

\section{REFERENCIAS BIBLIOGRÁFICAS}

Allard, M., Feyt, G., Fourny, M.C. y Talandier, M., 2014, "Raising awareness on mobility costs for households: a lever for changing residential choices and improving local governance? Experimentation in the French Alpine metropolitan area", en Transportation Research Procedia, vol. 4, 255 - 270.

Ascher, F., 2004, Los nuevos principios del urbanismo, Alianza Ensayo, Madrid.

Banister, D. y Hickman, R., 2006, "How to design a more sustainable and fairer built environment: transport and communications", en IEEE Proceedings of the ITS, vol. 153, núm. 4, 276-291.

Bazant, J., 2010, "Expansión urbana incontrolada y paradigmas de la planeación urbana", en Espacio Abierto, núm. 3, 475-503.

Baur, A., Thess, M., Kleinschmit, B. y Creutzig, F., 2014, "Urban Climate Change Mitigation in Europe: Looking at and beyond the Role of Population Density", en Journal of Urban Planning and Development, vol. 140, núm. 1, 04013003.

Bhatta, B., 2010, Analysis of urban growth and sprawl from remote sensing data, Springer, USA.

Blanco, I., Fleury, S. y Subirats, J., 2012, "Nuevas miradas sobre viejos problemas. Periferias urbanas y transformación social", en Gestión y Política Pública, volumen temático, 3-40.

Bruekner, J.K. y Fansler, D.A., 1983, "The economics of urban sprawl: Theory and evidence on the spatial sizes of cities", en Review Economics and Statistics, vol. 64, núm. 3, 479-482.

Brueckner, J.K. y Largey, A.G., 2008, "Social interaction and urban sprawl”, in Journal of Urban Economics, vol. 64, 18-34.

Bruton, M., 1985, Introduction to transportation planning, Hutchinson, London.

Bryant, C., Coppack, P. y Mitchell, C., 2000, “The city's countryside”, en Bunting, T. y Filion, P. (eds.), Canadian Cities in Transition, 2da ed. Oxford University Press, Don Mills, Ontario, 333-354.

Cervero, R. y Gorham, R., 1995, "Commuting in transit versus automobile neighborhoods", en Journal of the American Planning Association, vol. 61, 210-225.

Chen, C. y Mcknight, C., 2007, "Does the built environment make a difference? Additional evidence from the daily activity and travel behavior of homemakers 
living in New York City and suburbs", en Journal of Transport Geography, vol. $15,380-395$.

Coppack, P., Russwurm, L. y Bryant, C., 1988, Essays in Canadian Urban Process and Form 3: The Urban Field, University of Waterloo, Department of Geography, Waterloo.

Davies, W. y Murdie, R., 1993, "Measuring the social ecology of cities", en Bourne, L. y Ley, D. (eds.), The Changing Social Geography of Canadian Cities. McGill-Queen's University Press, Montreal, 52-75.

Da Gama-Torres, H., 2008, Social and environmental aspects of peri-urban growth in Latin American megacities. United Nations expert group meeting on population distribution, urbanization, integral migration and development, Nueva York.

Díaz, F., 2012, "Periferias urbanas y reconfiguración de las políticas urbanas en España”, en Gestión y Política Pública, volumen temático, 41-81.

Dupuy, G., 1995, Les territories de l'automobile, Anthropos-Economica, París.

Escolano, S. y Ortiz, J., 2005, "La formación de un modelo policéntrico de la actividad comercial en el Gran Santiago (Chile)", en Revista de Geografía Norte Grande, vol. 34, 53-64.

Friedmann, J., 2011, "Becoming urban: Periurban dynamics in Vietnam and China", en Pacific Affairs, vol. 84, 425-434.

Furuseth, O. y Lapping, M., 1999, Contested Countryside: The Rural-Urban Fringe in North America, Ashgate, Aldershot U.K.

García, J., 2008, "Incidencia en la movilidad de los principales factores de un modelo metropolitano cambiante", en EURE, vol. 34, núm. 101, 5-23.

García, J., 2010, "Urban sprawl and travel to work: the case of the metropolitan area of Madrid”, en Journal of Transport Geography, vol. 18, núm. 2, 197-213.

Giacobbe, N., Álvarez, A. y Pérez, L., 2009, “Aportes al estudio de la accesibilidad en el área metropolitana de Buenos Aires, el caso del municipio de Lanús", ponencia presentada en el XV Congreso Latinoamericano de Transporte Público y Urbano, 31 de marzo al 3 de abril, Buenos Aires, Argentina.

Goudie, D., 2002, “Zonal method for urban travel surveys: sustainability and sample distance from the CBD”, en Journal of Transport Geography, vol. 10, 287-301

Gutiérrez, J. y García, J., 2005, Movilidad por motivo de trabajo en la comunidad de Madrid, Universidad Complutense de Madrid, Madrid.

Haller, A., 2014, "The "sowing of concrete": Peri-urban smallholder perceptions of rural-urban land change in the Central Peruvian Andes", en Land Use Policy, vol. 38, 239-247.

Harvey, A., 1994, "Changing temporal perspectives and the Canadian metrópolis", en Frisken, F. (ed.), The Changing Canadian Metropolis: A Public Policy Perspective, vol. 1. Institute of Governmental Studies Press, Berkeley, Cal., 151-199. 
Harvey, R.O. y Clark, W., 1965, "The nature and economics of urban sprawl", en Land Economics, vol. 41, núm. 1, 1-9.

Hamidi, S. y Ewing, R., 2014, "A longitudinal study of changes in urban sprawl between 2000 and 2010 in the United States", en Landscape and urban planning, vol. 128, $72-82$.

Henry, E., 1998, "Regards sur la mobilité urbaine a Amerique latine", en Espaces et Societés, vol. 2, 52-58.

Hiernaux, D. y Lindón, A., 2004, "La periferia: voz y sentido en los estudios urbanos", en Papeles de Población, vol. 41, 101-123.

Ibeas, Á., González, F., Dellolio, L. y Moura, J., 2007, Manual de encuestas de movilidad (preferencias reveladas), Escuela Técnica Superior de Ingenieros de Caminos, Canales y Puertos de Santander, España.

INEGI, 2010, Compendio de criterios y especificaciones técnicas para la generación de datos e información de carácter fundamental, INEGI, México.

Lucatelli, S., 2011, Rurban: Rural-urban partnership for sustainable development, Working group on territorial cohesion and urban matters (TCUM), 15 December 2011, Bruselas.

Millward, H. y Spinney, J., 2009, "Time use and rurality: Canada 2005”, en Electronic International Journal of Time Use Research, vol. 6, 109-129.

Millward, H. y Spinney, J., 2011, "Time use, travel behavior, and the rural-urban continuum: Results from the Halifax STAR Project", en Journal of Transport Geography, vol. 19, 51-58.

Nechyba, T.J. y Walsh, R.P., 2004, “Urban Sprawl”, en Journal of Economic Perspectives, vol. 18, núm. 4, 177-200.

Newman, P.W.G. y Kenworthy, J.R., 1988, "The transport energy trade-off: fuel-efficient traffic versus fuel-efficient cities", en Transportation Research part A: Policy and Practice, vol. 22A, núm. 3, 163-174.

Nicolas, J.P., Pocheta, P. y Poimboeuf, H., 2003, “Towards sustainable mobility indicators: application to the Lyons conurbation", en Transport Policy, vol. 10, 197-208.

Norman, J., Maclean, H. y Kennedy, C., 2006, “Comparing High and Low Residential Density: Life-Cycle Analysis of Energy Use and Greenhouse Gas Emissions", en Journal of Urban Planning and Development, vol. 132, núm. 1, 10-21.

Obregón-Biosca, S.A., 2010, "Estudio comparativo del impacto en el desarrollo socioeconómico en dos carreteras: Eix Transversal de Catalunya, España y MEX120, México", en Economía Sociedad y Territorio, vol. X, núm. 32, 1-47.

Obregón-Biosca, S.A. y Bueno-Ortiz, C.O., 2015, "Dispersión urbana e integración funcional al núcleo central. Caso de estudio: Zona Metropolitana de Querétaro, México", en Gestión y Política Pública, vol. 24, núm. 2, 489-529. 
Obregón-Biosca, S.A., Romero Navarrete, J.A. y Betanzo Quezada, E. 2015a, "La movilidad en una zona metropolitana mexicana, caso de estudio: Querétaro, México", en Revista Transporte y Territorio, vol. 12, 167-197.

Obregón-Biosca, S.A., Romero-Navarrete, J.A., Mendoza-Sanchez, J.F. y Betanzo-Quezada, E., 2016, "Impact of Mobility Induced by Urban Sprawl: Case Study of the Querétaro Metropolitan Area”, en Journal of Urban Planning and Development, vol. 142, núm. 2, DOI: 10.1061/(ASCE)UP.1943-5444.0000295.

ONU, 2008, State of the World's Cities 2010/2011. Bridging the Urban Divide, United Nations Human Settlements Programme, Londres.

Ortúzar, J.D. y Willumsen, L., 2008, Modelos de Transporte, Universidad de Cantabria, España.

Pardo, C., 2005, "Salida de emergencia: reflexiones sociales sobre las políticas del transporte", en Universitas Psychologica, vol. 4, núm. 3, 271-284.

Pryor, R., 1968, "Defining the rural-urban fringe”, en Social Forces, vol. 47, 202-215.

Pucher, J. y Renne, J., 2005, "Rural mobility and mode choice: evidence from the 2001 National Household Travel Survey”, en Transportation, vol. 32, 165-186.

Putnam, R.D., 2000, Bowling Alone, Simon and Schuster, Nueva York.

Rahman, G., Alam, D. y Islam, S., 2008, "City growth with urban sprawl and problems of management", en Proccedings of 44th ISOCARP Congress Dalian, China, septiembre 19 al 23, International Society of City and Regional Planners and Urban Planning Society of China.

Ravetz, J., Fertner, C. y Sick Nielsen, Th.A., 2013, “The Dynamics of Peri-Urbanization", en Nilsson, K., Pauleit, S., Bell, S., Aalbers, C. y Sick-Nielsen, Th.A., (eds.), Peri-urban futures: Scenarios and models for land use change in Europe, DOI 10.1007/978-3-642-30529-0_2, Springer, Verlag Berlin Heidelberg.

SEDESOL, CONAPO e INEGI, 2012, Delimitación de las zonas metropolitanas de México 2010, Secretaría de Desarrollo Social-Consejo Nacional de PoblaciónInstituto Nacional de Geografía e Informática, México.

Smith, M.E., 1979, "Design of small sample home interview travel surveys", en Transportation Research Record, vol. 701, 29-35.

Sobrino, J., 2007, "Patrones de dispersión intrametropolitano en México", en Estudios Demográficos y Urbanos, vol. 22, núm. 3, 583-617.

Song, S., 1992, Monocentric and Polycentric Density Functions and Their Required Commutes, University of California Transportation Center, USA.

Sperandelli, D.I., Dupas, F.A. y Dias-Pons, N.A., 2013, "Urban sprawl, vacant land and green spaces dynamics on the metropolitan fringe of São Paulo, Brazil", en Journal of Urban Planning Development, vol. 139, 274-279.

Unikel, L., Ruiz, C. y Garza, G., 1976, El desarrollo urbano de México, Colegio de México, Centro de estudios económicos y demográficos, México. 
Valero, Á., 1984, "Movilidad espacial en Madrid", en Anales de Geografía de la Universidad Complutense, vol. 4, 207-225.

Webster, D., 2002, On the edge: Shaping the future of peri-urban Asia, Asia-Pacific Research Center, Stanford University, Stanford, CA.

Webster, D. y Muller, L., 2002, Challenges of peri-urbanization in the lower Yangtze region: the case of Hangzhou-Ninbo Corridor, Asia/Pacific Research Center, Stanford University, Stanford, CA.

Wehrwein, G., 1942, "The rural-urban fringe", en Economic Geography, vol. 18, 217-228.

Zasada, I., Fertner, C., Piorr, A. y Nielsen, T., 2011, "Peri-urbanisation and multifunctional adaptation of agriculture around Copenhagen", en Geografisk Tidsskrift-Danish Journal of Geography, vol. 111, núm. 1, 59-72.

Zhang, B., 2004, Study on Urban Growth Management in China, Xinhua Press, Beijing.

Zhang, L., 2010, "Do Freeway Traffic Management Strategies Exacerbate Urban Sprawl? The Case of Ramp Metering", in Transportation Research Record, vol. 2174, 99-109.

\section{Resumen CURRICUlar DEL AUTOR}

\section{Saúl Antonio Obregón Biosca}

Obtuvo el doctorado en el programa de Transporte y Ordenación del Territorio, suficiencia investigadora en urbanística y la maestría en la Universidad Politécnica de Cataluña (UPC), ingeniero civil por la Universidad Autónoma de Querétaro (UAQ). Desde 2009 trabaja como profesor-investigador en la División de Estudios de Posgrado de la Facultad de Ingeniería de la UAQ, en donde coordinó la Maestría en Vías Terrestres y Movilidad desde el año 2009 al 2018. Es miembro del Sistema Nacional de Investigadores de México en el Nivel 2 y de la Sociedad Americana de Ingenieros Civiles. En 2016 obtuvo el Premio Nacional "José Carreño Romaní 2016" otorgado por la AMIVTAC- SCT. Su actividad como investigador se ha centrado en la ingeniería de redes de infraestructura en el territorio, en dos ámbitos: en primer lugar, la infraestructura de transporte en la ordenación del territorio, el segundo, movilidad metropolitana. Recientemente su investigación ha abordado el factor humano dentro de la seguridad vial. Dirección electrónica: saul.obregon@uaq.mx

Artículo recibido el 28 de septiembre de 2015 y aprobado el 19 de julio de 2018. 